The Astrophystcal Journal, Vol. 154, October 1968

\title{
ANALYSIS OF $F$ AND G SUBDWARFS. III. AN ABUNDANCE ANALYSIS OF THE SUBDWARF $\mu$ CASSIOPEIA
}

\author{
JUdith G. COHEN* \\ Smithsonian Astrophysical Observatory and Harvard College Observatory \\ Received A ugust 23, 1967; revised A pril 8, 1968
}

\begin{abstract}
Equivalent widths of lines with $\lambda>4600 \AA \AA$ were measured from a set of $4.5 \AA \mathrm{mm}^{-1}$ Palomar plates and were used in performing a model-atmosphere abundance analysis of the mild subdwarf $\mu$ Cas. Comparison with the Sun was made by computing abundances from individual solar lines using the same set of transition probabilities as was used for the subdwarf Results of this analysis show that the iron-peak elements appear to be deficient by a factor of 4 , while the $a$ process in $\mu$ Cas elements may be enhanced with respect to these elements.
\end{abstract}

Because of its moderately large ultraviolet excess, $\delta(U-B)=0.16, \mu$ Cas has been identified as a subdwarf by Sandage and Eggen (1959). This star is also an astrometric binary (Lippincott and Wyckoff 1964), although only a crude measurement of the separation of the two components is as yet available (Wehinger and Wyckoff 1966).

Since $\mu$ Cas has a large parallax, $\pi=0 \prime \prime 127 \pm 0$ ".003 (Lippincott and Wyckoff 1964), it can be accurately placed on the $\mathrm{H}-\mathrm{R}$ diagram if its effective temperature is known (Strom, Cohen, and Strom 1967, Paper I of this series). Strom and Strom (1967) have determined the effective temperature to be $5000^{\circ} \pm 150^{\circ} \mathrm{K}$ from a comparison of scans made in the region 5000-8000 $\AA$ with the predictions of model atmospheres.

Using Wallerstein's (1962) correlation between $\delta(U-B)$ and metal content, we deduce that $\mu$ Cas lies less than $0.1 \mathrm{mag}$ above the theoretical zero-age main sequence (Faulkner and Iben 1966) appropriate to its metal content. We do not consider this difference to be significant, since either small changes in the convective efficiencies or a slight error in the computed bolometric magnitude can account for such a difference.

Unfortunately, no spectroscopic information on the surface gravity is available, and, since the separation of the two components has not been measured accurately, the mass of the primary is uncertain. We do not expect and do not apparently find $\mu$ Cas to have evolved from the main sequence over a time scale comparable to the age of the Galaxy. By using the quasi-homology relations of Faulkner (1967), the computed evolutionary tracks of Faulkner and Iben (1966), we can therefore choose $(5000,4.4,0.03,0)$ for our initial model. The system for designating models is $\left(T_{\text {eff }}, \log g, Z / Z \odot, v_{t}\right)$, where $T_{\text {eff }}$ is effective temperature, $g$ is surface gravity, $Z / Z \odot$ is metal content relative to the Sun, and $v_{t}$ is microturbulent velocity in $\mathrm{km} \mathrm{sec}^{-1}$. All models satisfy radiative equilibrium and LTE and have been obtained with Strom's programs as described in Strom and Avrett (1965) and in Paper II (Cohen and Strom 1967). No detailed analysis of $\mu$ Cas has been previously carried out; ${ }^{1}$ hence the value for $Z / Z \odot$ is a guess, and the zero microturbulent velocity was chosen to correspond to the results of Wallerstein (1962) and Aller and Greenstein (1960) for Population II stars.

We obtained the equivalent widths from two $4.5 \AA \mathrm{mm}^{-1}$ Palomar plates and calibration wedges exposed by Dr. J. B. Oke. The spectra were traced on the David Mann

* Currently at Department of Astronomy, California Institute of Technology.

${ }^{1}$ At the time this paper was submitted, a differential curve-of-growth analysis was published by Catchpole, Pagel, and Powell (1967). The results of this work are in substantial agreement with the model-atmosphere analysis reported here as shown in Table 1. 
microphotometer of the Harvard College Observatory. The transformation of the transmission versus position data into intensities versus wavelength and then to equivalent widths was carried out on a CDC 6400 computer. Approximately 250 lines were chosen (in the wavelength regions 4650-4900 $\AA$ and 5100-6250 $\AA$ ) for analysis, and a table of equivalent widths is available from the author upon request. Errors of no more than \pm 20 per cent in $W_{\lambda}$ are expected.

Lines falling at shorter wavelengths have been used in most previous detailed analyses. As a result, many errors in the normalization of the scales of transition probabilities for lines with $\lambda>5000 \AA$ have not yet been discussed. Furthermore, since we have used a selection of transition probabilities from the recent literature, the absolute scale of our $g f$-values is in many cases different from that used by Goldberg, Müller, and Aller (1960) (hereinafter called "GMA") in their study of the Sun. Since we wish to obtain abundances in $\mu$ Cas relative to the Sun as well as to determine the absolute metal-tohydrogen ratios, the normalization of the $g f$-values is critical. We have therefore adopted a procedure that preserves the advantages of a detailed model atmospheric analysis (Strom, Gingerich, and Strom 1966) and also insures the validity of comparisons with the solar abundances. For each element, the solar equivalent widths for lines corresponding to those observed in $\mu$ Cas were obtained from the revised Rowland tables (Moore, Minnaert, and Houtgast 1966). If more than ten lines of an element were observed in $\mu$ Cas, a random selection from the subdwarf lines was made. We then used the same transition probabilities as had been used for the subdwarf analysis and performed the identical analysis for the Sun, using the Utrecht reference model (URM) (Heintze, Hubenet, and de Jager 1964) assuming a constant microturbulent velocity of $1.4 \mathrm{~km} \mathrm{sec}^{-1}$ throughout the solar atmosphere. Although the solar equivalent widths in the revised Rowland tables are reported for the center of the disk, we have treated them as if they were identical to those that would be obtained from the integrated disk; the errors thus introduced will generally not be large, as can be seen by comparing the widths for various values of $\cos \theta$ given by Withbroe (1967). We shall call the abundances obtained using our transition probabilities, the equivalent widths determined as above, and the URM the new "reference-Sun" abundances.

Sometimes the same abundance for a given element is not deduced from each ionization stage even though the photospheric structure for the Sun is rather well known in the region of formation of the weak lines. We therefore assume that the difference in abundance between two ionization stages for the solar analysis is caused solely by errors in the scale of the $g f$-values. We then proceed to choose the appropriate model for $\mu$ Cas by adjusting the atmospheric parameters until the same difference in abundance is achieved for the two ion states in question. We stress that the abundances listed in the column "Reference Sun" of Table 1 are not suggested revisions of the GMA solar abundances. Rather, they indicate (1) differences and possible errors in the absolute scale of the transition probabilities for this particular wavelength region and for this selection of lines, (2) possible errors in the model solar atmosphere, or (3) systematic errors in the solar equivalent widths given by Moore, et al. (1966). In all but a few cases, the first contribution is undoubtedly the largest.

The final model for $\mu$ Cas $(5100,4.2,0.2,3)$ was chosen to produce the correct ionization equilibrium and a constant deduced abundance as a function of both $W_{\lambda}$ and $\chi$, the excitation potential of the lower level, for $\mathrm{Fe}$ I lines. Note the rather high value of the microturbulent velocity $\left(v_{t}=3 \mathrm{~km} \mathrm{sec}{ }^{-1}\right)$, which disagrees with previous results on other subdwarfs (Wallerstein 1962) and the result of $v_{t}=1.5 \mathrm{~km} \mathrm{sec}^{-1}$ obtained by Catchpole et al. (1967). A possible source of this disagreement is discussed in Paper II. We see in Figure 1, which shows deduced abundance as a function of equivalent width for $\mathrm{Fe} \mathrm{I}$ lines, that the final model gives quite consistent results. We have eliminated all lines that fall on the damping part of the curve of growth in making this plot. Owing to the lack of accurate damping constants (ten times the classical value was used through- 
out this analysis), lines with equivalent widths greater than $160 \mathrm{~m} \AA$ were not used in the abundance analysis.

No wavelength dependence greater than 0.15 in the logarithm of the average abundance for lines in the two regions 4650-4900 $\AA$ and 5100-6250 $\AA$ has been observed for elements having a sufficient number of lines to make such a test (Fe I, Ti I, Ni I, and V I). This suggests that the reference-Sun and GMA abundances should agree for these elements. We shall see that this holds, except for titanium.

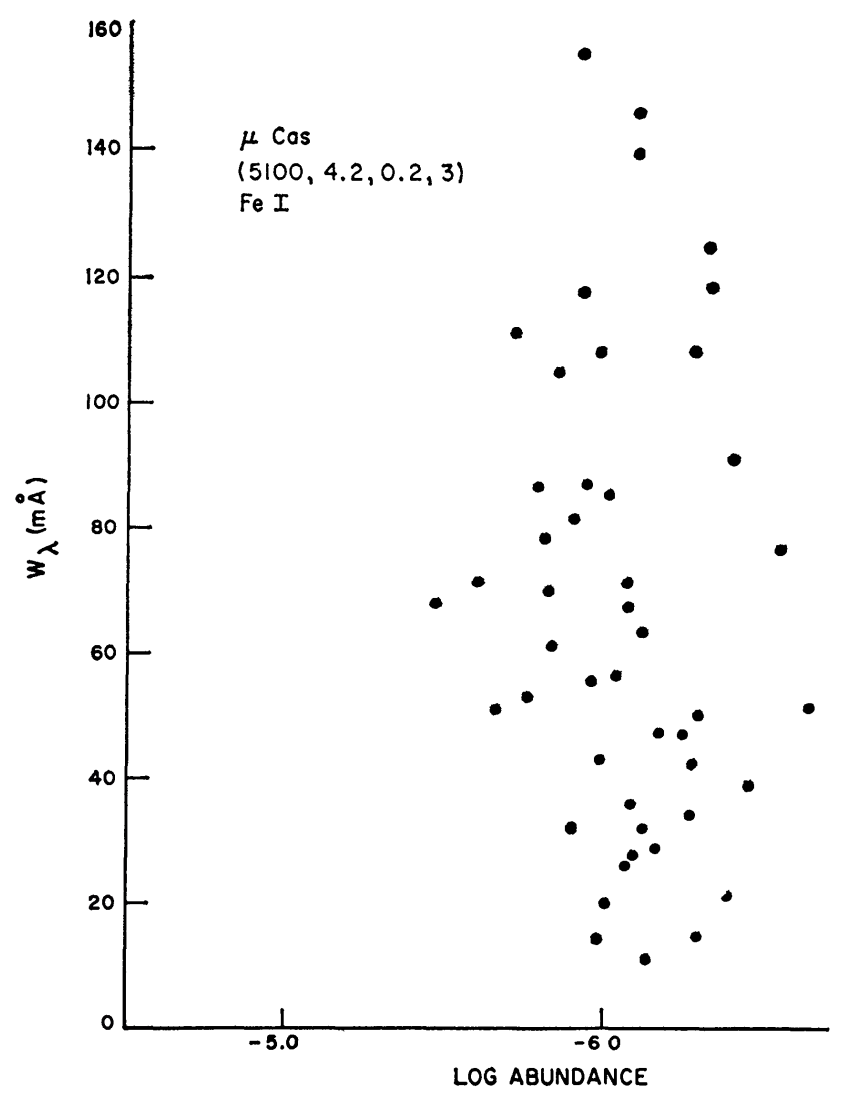

FIg 1.-Deduced abundance for $\mathrm{Fe}$ I lines in $\mu$ Cas as a function of equivalent width for the model $(5100,4.2,0.2,3)$.

In Table 1 we present the abundances $\log \left(N_{\mathrm{el}} / N_{\mathrm{H}}\right)$ for $\mu$ Cas for the final model and the number of lines in $\mu$ Cas for each element, the GMA and the reference-Sun abundances, and the number of lines that were used to construct the reference-Sun abundances. The results of Catchpole et al. (1967) are also displayed, normalized to our $\mathrm{Fe}$ I abundance. Some comments on the individual elements are given below:

Ba II.-We have used the Corliss and Bozman (1962) transition probabilities (hereinafter abbreviated as "CB"). Garstang and Hill's (1966) $g f$-values have the same ratio for these two lines, although the absolute scale is different.

C I.-All the lines in $\mu$ Cas are quite weak. The only line in the long-wavelength region under study here gives an abundance of -3.5 . It is possible, though not probable, that the three lines measured near $4800 \AA$ are misidentified. However, we note that in Groombridge 1830, a slightly more metal-deficient subdwarf of almost the same $T_{\text {eff }}$, these three carbon lines are also enhanced (Greenstein 1966). From comparison of CH 
and C I abundances, we feel that the $g f$-scale (Griem 1964) is satisfactorily normalized.

Ce II.- The value given here for the Sun is that of Allen (1963).

$\mathrm{Cr}$ I and Cr II.- Note the poor ionization equilibrium obtained for the Sun, which seems to indicate an error in the normalization of the absolute $g f$-values for these two ionization stages.

$\mathrm{Cu}$ I.-There appears to be a large difference between the $g f$-scale of $\mathrm{CB}$ and that used by GMA for the two lines observed in $\mu$ Cas, as has been noticed by Aller (1965).

Fe I and Fe Ir.-The agreement between our abundance and that of GMA is good.

TABLE 1

AvERAGE ABUndanCES FOR $\mu$ CAS FOR THE FINAL MODEL $(5100,4.2,0.01,3)$

- COMPARED to CATCHPOle, PAGEL, AND POWELl (1967)

\begin{tabular}{|c|c|c|c|c|c|c|}
\hline Element & $\begin{array}{l}\text { Reference } \\
\text { Sun }\end{array}$ & $\begin{array}{l}\text { Number of } \\
\text { Lines }\end{array}$ & GMA & $\begin{array}{l}\text { Subdwarf } \\
\text { Abundance }\end{array}$ & $\begin{array}{l}\text { Number of } \\
\text { Lines }\end{array}$ & $\begin{array}{l}\text { Abundances of } \\
\text { Catchpole } \text { et al }\end{array}$ \\
\hline 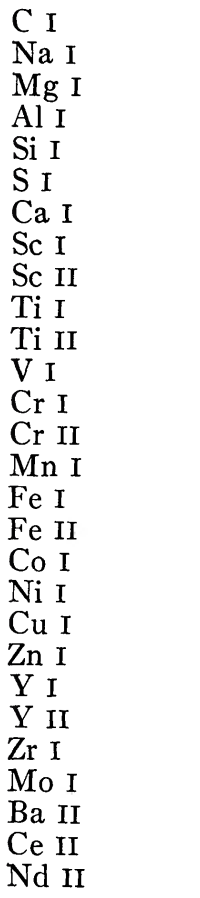 & $\begin{array}{rr}3 & 21 \\
5 & 70 \\
4 & 58 \\
6 & 12 \\
4 & 60 \\
5 & 06 \\
5 & 97 \\
8 & 69 \\
8 & 80 \\
7 & 67 \\
7 & 14 \\
8 & 29 \\
6 & 92 \\
6 & 59 \\
7 & 07 \\
5 & 45 \\
5 & 34 \\
7 & 77 \\
6 & 33 \\
8 & 15 \\
5 & 32 \\
8 & 94 \\
9 & 49 \\
8 & 71 \\
10 & 92 \\
9 & 58 \\
9 & 28 \\
-8 & 29\end{array}$ & $\begin{array}{r}4 \\
5 \\
2 \\
1 \\
9 \\
3 \\
10 \\
6 \\
28 \\
18 \\
4 \\
10 \\
8 \\
6 \\
9 \\
9 \\
9 \\
11 \\
14 \\
2 \\
1 \\
1 \\
2 \\
4 \\
1 \\
2 \\
3 \\
4\end{array}$ & $\begin{array}{rl}-3 & 28 \\
5 & 70 \\
4 & 60 \\
5 & 80 \\
4 & 70 \\
4 & 50 \\
5 & 85 \\
9 & 18 \\
7 & 32 \\
8 & 30 \\
8 & 30 \\
6 & 64 \\
5 & 10 \\
5 & 43 \\
7 & 36 \\
6 & 09 \\
6 & 94 \\
7 & 60 \\
9 & 75 \\
. & 79 \\
9 & 77 \\
10 & 10 \\
9 & 90 \\
10 & 4 \\
-10 & 5\end{array}$ & $\begin{array}{rr}-3 & 01 \\
6 & 46 \\
5 & 17 \\
6 & 81 \\
4 & 97 \\
4 & 90 \\
6 & 44 \\
9 & 35 \\
9 & 27 \\
8 & 07 \\
7 & 68 \\
8 & 62 \\
7 & 60 \\
7 & 02 \\
7 & 93 \\
6 & 02 \\
5 & 90 \\
8 & 30 \\
7 & 10 \\
8 & 95 \\
6 & 10 \\
9 & 62 \\
10 & 10 \\
9 & 05 \\
11 & 96 \\
10 & 41 \\
9 & 88 \\
-8 & 87\end{array}$ & $\begin{array}{r}4 \\
5 \\
2 \\
1 \\
9 \\
3 \\
10 \\
6 \\
10 \\
23 \\
4 \\
23 \\
20 \\
7 \\
17 \\
62 \\
9 \\
11 \\
28 \\
2 \\
1 \\
1 \\
4 \\
4 \\
1 \\
2 \\
3 \\
5\end{array}$ & $\begin{array}{rl}-6 & 34 \\
4 & 88 \\
6 & 9 \\
4 & 99 \\
6 & 11 \\
6 & 11 \\
9 & 22 \\
8 & 02 \\
7 & 48 \\
8 & 81 \\
7 & 57 \\
\dot{7} & 92 \\
5 & 91 \\
\dot{8} & 33 \\
6 & 88 \\
6 & 02 \\
5 & 78 \\
10 & 05 \\
8 & 76^{*} \\
10 & 3 \dot{35} \\
-8 & 61^{*}\end{array}$ \\
\hline
\end{tabular}

* Upper limit owing to weakness of lines observed by Catchpole et al (1967)

Nd II.-The value given here for the Sun is that of Allen (1963).

$\mathrm{Ni}$ I. - The same shift is found for lines in the visible, as discussed in Paper II. We used Corliss' (1965) $g f$-values.

S I.-The solar lines and those in $\mu$ Cas are quite weak, so that the difference between the reference-Sun abundance, obtained with $g f$-values given by Kohl (1964), and the GMA abundance may not be meaningful.

Sc.-There appears to be a difference in the absolute normalization of the $\mathrm{CB}$ and the GMA scales of transition probabilities.

Ti.-We have used $\mathrm{CB}$ values for Ti I and two of the four Ti II lines. Transition probabilities for the other two Ti II lines were taken from Tatum (1961). His scale appears to differ from the CB normalization by $0.5-0.8$ in the logarithm. 
Y.-There appears to be an error in the normalization of the CB scale. We have noticed a similar problem for Y I and Y II lines in the visual.

$\mathrm{Zr}$ I. - These lines are so weak that the identifications are doubtful (the largest $W_{\lambda}$ of the four lines in the Sun is $3.5 \mathrm{~m} \AA$ ). If the identifications are correct, there must be a large change in normalization. We have used the CB scale for this element.

Note that for cerium, molybdenum, neodymium, and zinc no accurate partition functions were available. The same value for each element has been adopted in analyzing $\mu$ Cas and the Sun. Thus, errors in the partition function will appear as errors in the absolute value of the abundances if the atom is partially ionized, but we feel that relative abundances of the subdwarf as compared to the reference Sun should be largely unaffected.

Such uncertainties in the absolute scale of transition probabilities make it difficult to ascertain the absolute deficiencies of elements representing the various processes of nucleosynthesis. We have used the reference-Sun abundances deduced with the same set of transition probabilities as were used in the analysis of $\mu$ Cas to construct Table 2, which displays the average deficiencies of $a-, e-$, and $s$-process elements. Since the abundance of zirconium is so uncertain, it has been omitted in obtaining the average, as have

TABLE 2

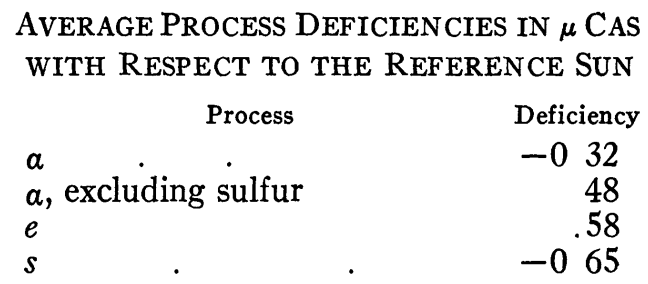

all elements with only one observed line. Although the scatter is larger than any of the differences between the average deficiencies, it is clear that metals in $\mu$ Cas are deficient by about a factor of 4 , and it is possible that the a-process elements are slightly enhanced, while the $s$-process elements are slightly more deficient than the iron-peak elements. The agreement between our results and those of Catchpole et al. (1967), using a differential curve-of-growth analysis, is rather striking considering that the equivalent widths and model parameters were separately determined for each investigation.

We note that errors in the absolute calibration of Vega affect our choice of effective temperature for $\mu$ Cas from the scans. Calculations of ionization equilibrium at various values of $T_{\text {eff }}$ and $\log g$ show that in this temperature range, if we increase $T_{\text {eff }}$ by $250^{\circ} \mathrm{K}$, we must increase $\log g$ by 0.4 to preserve ionization equilibrium. Thus, in order to obtain agreement between the spectroscopic determination of $\log g=4.2$ for $\mu$ Cas and a determination based on the mass-luminosity relationship given in Catchpole et al. (1967) of 4.4 , we must have underestimated $T_{\text {eff }}$ as determined from the scans by $\sim 150^{\circ} \mathrm{K}$. Recent results on the recalibration of Vega by Hayes (1967) suggest that the slope of that star's Paschen continuum must be increased. By using this new calibration, the values of $T_{\text {eff }}$ for the subdwarfs must then be increased by about $200^{\circ} \mathrm{K}$, in close agreement with the spectroscopic conclusions.

The neglect of convection is a possible source of error in this analysis. However, we expect that, in this mildly metal-deficient subdwarf, the flattening of the temperature gradient will occur only at depths greater than the line-forming region. It is possible that our difficulties with zirconium and carbon are due to the change in temperature structure at the comparatively large optical depths, where these very weak lines are formed.

In summary, the problems of line blending in stars with $T_{\text {eff }}<5500^{\circ} \mathrm{K}$ force one to 
use spectral lines at longer wavelengths for detailed abundance analyses. Uncertainties in the calibration of $g f$-values for $\lambda>5000 \AA$ are large and cannot be neglected. Bearing in mind these differences between our transition probabilities and those used by GMA, we obtain a metal deficiency of a factor of 4 for $\mu$ Cas as compared with the Sun. Comparison of the results of Catchpole et al. (1967) and the present analysis for $\mu$ Cas gives convincing evidence of the validity of the methods employed in each investigation and the accuracy of the final results.

I would like to thank Dr. J. B. Oke for the use of his plates, Dr. W. Parkinson of Harvard College Observatory for permission to use the David Mann microphotometer, Dr. Stephen Strom and Mr. R. Kurucz for the use of their programs and for helpful discussion.

This research has been supported in part by grant NGR 22-024 001 from the National Aeronautics and Space Administration.

\section{REFERENCES}

Allen, C. W. 1963, Astrophysical Quantities (2d ed ; London: Athlone Press).

Aller, L. H. 1965, Adv. Astr and $A p, 3,1$.

Aller, L. H., and Greenstein, J L. 1960, Ap. J. Suppl., 5, 139.

Catchpole, R M., Pagel, B E. J., and Powell, A. L. T. 1967, M.N.R.A S., 136, 403

Cohen, J. G., and Strom, S. E. 1967, Ap. J., 151, 623 (Paper II).

Corliss, C. H 1965, N.BS. J. Res. , 69A, 87.

Corliss, C. H, and Bozman, W. R. 1962, N.B S. Monog , No. 53 (Washington, D.C : Government Printing Office).

Faulkner, J. 1967, Ap. J., 147, 617.

Faulkner, J , and Iben, I., Jr, 1966, $A p J, 144,995$.

Garstang, R. H., and Hill, S J. 1966, Pub. A SP, 78, 70.

Goldberg, L., Müller, E. A , and Aller, L. H 1960, Ap J.Suppl., 5, 1.

Griem, M R 1964, Plasma Spectroscopy (New York: McGraw-Hill).

Greenstein, J. L. 1966, unpublished

Hayes, D. 1967, unpublished Ph D thesis, University of California at Los Angeles

Heintze, J. R. W., Hubenet, M., and de Jager, C. 1964, Smithsonian Ap Obs. Spec. Rept No. 167, p 239.

Kohl, K. 1964, Zs.f. Ap., 60, 115

Lippincott, S L., and Wyckoff, S. 1964, $A J ., 69,471$.

Moore, C. E., Minnaert, M. G. J, and Houtgast, J. 1966, N B S Monog, No 61 (Washington, D C.: Government Printing Office).

Sandage, A. R., and Eggen, O J. 1959, M.N R.A S., 119, 278.

Strom, S. E., and Avrett, E. M 1965, Ap. J. Suppl, 12, 1.

Strom, S E., Cohen, J G., and Strom, K M. 1967, Ap.J., 147, 1038 (Paper I).

Strom, S. E., Gingerich, O. J., and Strom, K M 1966, $A$ p. J., 146, 880.

Strom, S. E., and Strom, K. M. 1967, Ap.J , 150, 501.

Tatum, J. B. 1961, Com. U. London Obs., No 41.

Wallerstein, G. 1962, Ap.J.Suppl., 6, 407

Wehinger, P., and Wyckoff, S. 1966, A.J., 71, 185.

Withbroe, G. L. 1967, Ap.J., 147, 1117.

Copyright 1968 The University of Chicago Printed in U.S A 